\title{
Importance of balloon valvuloplasty before TAVI to prevent embolization
}

Omission of balloon aortic valvuloplasty before implanting a balloon-expandable aortic valve shortens procedure time and lowers contrast volume, but is associated with an increase in cerebral ischaemic lesions. This finding comes from a nonrandomized, single-centre study conducted in Hamburg, Germany.

A total of 87 consecutive, high-risk patients underwent transcatheter aortic valve implantation (TAVI) with a balloonexpandable valve and either received $(n=32)$ or did not receive $(n=55)$ prior balloon valvuloplasty. Procedural success was $93.5 \%$ and $98.2 \%$ in each group, respectively. As expected, the duration of the procedure $(69.2 \mathrm{~min}$ vs $58.7 \mathrm{~min}$; $P=0.008)$ and the volume of contrast agent ( $97.0 \mathrm{ml}$ vs $69.8 \mathrm{ml} ; P=0.004$ ) were significantly higher in patients who received balloon valvuloplasty compared with those who did not. However, diffusion-weighted MRI performed
2-7 days after TAVI revealed that patients who did not receive balloon valvuloplasty had a significantly higher volume of cerebral ischaemic lesions $\left(235.5 \mathrm{~mm}^{3}\right.$ vs $\left.89.5 \mathrm{~mm}^{3} ; P=0.01\right)$.

Whether this increased rate of cerebral ischaemic lesions translates into a higher rate of stroke remains to be established. Nevertheless, the investigators comment that "cerebral ischaemic lesions potentially deteriorate neurocognitive function and should therefore be avoided. Cerebral embolic protection devices may be of specific value for TAVI without balloon aortic valvuloplasty using a balloon-expandable valve."

Gregory B. Lim

Original article Bijuklic, K. et al. Increased risk of cerebral embolization after implantation of a balloon-expandable aortic valve without prior balloon valvuloplasty. JACC Cardiovasc. Interv. doi:10.1016/ j.jcin.2015.07.013 